\title{
Elevated hepatic expression of H19 long noncoding RNA contributes to diabetic hyperglycemia
}

\author{
Na Zhang, ${ }^{1,2}$ Tingting Geng, ${ }^{1,3}$ Zhangsheng Wang, ${ }^{1,4}$ Ruling Zhang, ${ }^{1,5}$ Tiefeng Cao, ${ }^{1,6}$ \\ Joao Paulo Camporez, ${ }^{7}$ Shi-Ying Cai, ${ }^{7}$ Ya Liu, ${ }^{1,8}$ Luisa Dandolo, ${ }^{9}$ Gerald I. Shulman, ${ }^{7}$ \\ Gordon C. Carmichael, ${ }^{2}$ Hugh S. Taylor,' and Yingqun Huang
}

'Department of Obstetrics, Gynecology, and Reproductive Sciences, Yale University School of Medicine, New Haven, Connecticut, USA. ${ }^{2}$ Department of Genetics and Genome Sciences, University of Connecticut Health Center, Farmington, Connecticut, USA. ${ }^{3}$ Department of Endocrinology, First Affiliated Hospital of Xi'an Jiaotong University School of Medicine, Xi'an, China. ${ }^{4}$ Department of Cardiology, Fifth People's Hospital of Shanghai, Fudan University, Shanghai, China. ${ }^{5}$ Department of Gastroenterology, Shanghai General Hospital, Shanghai Jiaotong University School of Medicine, Shanghai, China. ${ }^{6}$ Department of Gynecology and Obstetrics, First Affiliated Hospital of Sun Yat-Sen University, Guangzhou, Guangdong, China. 'Department of Internal Medicine, Yale University School of Medicine, New Haven, Connecticut, USA. ${ }^{8}$ Department of Veterinary Medicine, College of Animal Science and Technology, Anhui Agricultural University, Hefei, Anhui, China. ${ }^{9}$ Department of Cenetics and Development, Inserm U1016, Institut Cochin, Paris, France.

Excessive hepatic glucose production (HCP) contributes significantly to the hyperglycemia of type 2 diabetes; however, the molecular mechanism underlying this dysregulation remains poorly understood. Here, we show that fasting temporally increases the expression of H19 long noncoding RNA (IncRNA) in nondiabetic mouse liver, whereas its level is chronically elevated in diet-induced diabetic mice, consistent with the previously reported chronic hepatic $\mathrm{H} 19$ increase in diabetic patients. Importantly, liver-specific H19 overexpression promotes HCP, hyperglycemia, and insulin resistance, while $\mathrm{H} 19$ depletion enhances insulin-dependent suppression of HGP. Using genomewide methylation and transcriptome analyses, we demonstrate that $\mathrm{H} 19$ knockdown in hepatic cells alters promoter methylation and expression of $\mathrm{Hnf4a}$, a master gluconeogenic transcription factor, and that this regulation is recapitulated in vivo. Our findings offer a mechanistic explanation of IncRNA H19's role in the pathogenesis of diabetic hyperglycemia and suggest that targeting hepatic $\mathrm{H} 19$ may hold the potential of new treatment for this disease.

Authorship note: NZ and TC contributed equally to this work.

Conflict of interest: The authors have declared that no conflict of interest exists.

Submitted: February 1, 2018

Accepted: April 19, 2018

Published: May 17, 2018

\section{Reference information:} JCI Insight. 2018;3(10):e120304. https://doi.org/10.1172/jci. insight.120304.

\section{Introduction}

Type 2 diabetes mellitus (T2D) is a disease of impaired glucose homeostasis, which is characterized by elevated blood glucose levels (hyperglycemia) and insulin resistance. Chronic hyperglycemia causes damage to major organs, including the blood vessels, heart, liver, brain, kidneys, and eyes, contributing heavily to the health and economic burden associated with this illness. As the main organ of endogenous glucose production (EGP), the liver is especially critical in maintaining blood glucose levels. It is widely accepted that excessive hepatic glucose production (HGP) is the major contributing factor in the hyperglycemia of T2D (reviewed in refs. 1, 2). In healthy individuals, the increase in HGP has been ascribed to enhanced glycogenolysis and gluconeogenesis, leading to the production of glucose-6-phosphate, which is subsequently dephosphorylated by glucose-6-phosphatase (G6PC) to become glucose, which is then released into the circulation. HGP is activated by glucagon during the fasted state and inhibited by insulin during the fed state. The regulatory pathways involved are characterized by an intricate web of transcriptional factors and coactivators that regulate the expression of key gluconeogenic enzymes, including phosphoenolpyruvate carboxykinase (PEPCK) and G6PC; both are rate-limiting enzymes that catalyze the first and last steps of gluconeogenesis reaction, respectively $(1,2)$. During fasting, the expression of the transcriptional coactivator PGC- $1 \alpha$ is increased, leading to activation of the entire gluconeogenesis program (3). Importantly, the hepatocyte nuclear factor $4 \alpha(\mathrm{HNF} 4 \mathrm{~A})$ has been shown to be absolutely required for the PGC-1 $\alpha$-induced expression of $P c k 1$ and $G 6 p c(3,4)$. The transcriptional activation involves cooperative 
binding of PGC-1 $\alpha$ and HNF4A to well-characterized cis-elements on the promoters of Pck1 and G6pc (3, 4). Consistent with impaired insulin-mediated suppression of HGP in T2D, the expression of Hnf4a, Pck1, and $G 6 p c$ was increased in the liver of multiple animal models of diabetes (5-7).

The evolutionarily conserved imprinted H19, together with its coregulated Igf2, plays an important role in embryo development and growth control and has been associated with human genetic disorders. H19 is highly expressed in fetal tissues but strongly downregulated after birth - except in a few tissues, including skeletal muscle and heart. $H 19$ encodes a multifunctional long noncoding RNA (lncRNA; hereafter called H19) that acts both in the nucleus and in the cytoplasm (8). We have recently found that H19 modulates DNA methylation genome-wide, in part through interaction with $S$-adenosylhomocysteine hydrolase (SAHH) in mouse skeletal muscle and human cancer cells $(9,10)$. SAHH is the only mammalian enzyme capable of hydrolyzing $S$-adenosylhomocysteine (SAH), which is a potent feedback inhibitor of $S$-adenosylmethionine-dependent (SAM-dependent) methyltransferases, including DNA methyltransferases. H19 binds to SAHH and inactivates its enzymatic activity. This causes accumulation of SAH and inhibition of DNA methyltransferases, leading to decreased DNA methylation $(9,10)$. Importantly, H19 is expressed at low but appreciable levels in adult livers (11). Interestingly, its expression is chronically elevated in T2D patients with unknown biological significance (12). We hypothesized that the chronic increase of hepatic H19 expression may contribute to abnormal glucose production and, hence, hyperglycemia in T2D patients.

In this report, we show chronically increased H19 expression in diet-induced diabetic mouse liver, recapitulating observations from human patients (12). Importantly, liver-specific H19 overexpression promotes HGP, hyperglycemia, and insulin resistance, whereas H19 deletion leads to enhanced insulin-dependent suppression of HGP. Mechanistically, we provide evidence that an H19-induced alteration in promoter methylation and expression of Hnf4a may contribute to the underlying mechanism.

\section{Results}

Hepatic H19 expression is chronically increased in diabetic mice. To test whether increased hepatic H19 expression seen in T2D human patients (12) could be recapitulated in a mouse model, we treated mice with a high-fat diet (HFD). Compared with normal chow-fed (NC-fed) mice, the HFD-fed mice gained more weight (Figure 1A), had an elevated fasting glucose (Figure 1B), and became glucose intolerant (Figure 1C). These mice also displayed increased hepatic expression of H19 (Figure 1D). These results demonstrate a positive relationship between increased hepatic $\mathrm{H} 19$ expression and impaired glucose homeostasis.

Liver-specific H19 overexpression promotes HGP, hyperglycemia, and insulin resistance. To determine whether increased hepatic H19 expression is associated with impaired glucose metabolism, a tissue-specific H19 gain-of-function mouse model was used. Thus, H19 was overexpressed in the liver (Figure 1E) using a recombinant adeno-associated virus serotype 8 (AAV8) vector shown to have superior tropism for liver $(13,14)$. Liver-specific H19 overexpression was achieved by i.v. administration of AAV-H19 that expresses mouse full-length $\mathrm{H} 19$ driven by a liver-specific promoter, thyroxin-binding globulin (TBG) $(13,14)$. The lack of differential H19 expression in other tissues between the AAV-Vec- and AAV-H19injected mice further confirmed liver-specific H19 overexpression (Supplemental Figure 1; supplemental material available online with this article; https://doi.org/10.1172/jci.insight.120304DS1). The H19 gain-of-function mice showed increase in both fasting glucose (Figure 1F) and insulin (Figure 1G) levels. Pyruvate tolerance tests (PTT, a readout for HGP; refs. 15, 16) revealed higher glucose levels following pyruvate injection (Figure 1H). The H19-overexpressed mice also were glucose intolerant (Figure 1I) and insulin resistant (Figure 1J). Collectively, these results suggest that an elevated level of H19 in the liver augments HGP and impairs glucose homeostasis.

H19 deletion enhances insulin-dependent suppression of HGP. To further characterize H19's role in HGP and glucose metabolism, we used a whole-body H19-KO mouse model $(17,18)$. The KO mice showed an overgrowth phenotype and skeletal muscle hyperplasia and hypertrophy $(17,18)$, consistent with the role of the imprinted H19-Igf2 locus in embryo development and growth regulation (8). However, the metabolic phenotype of the KO mice has not been previously documented. To determine effects of H19 loss of function in HGP, hyperinsulinemic-euglycemic clamp studies were performed. The glucose infusion rate (GIR) in the KO mice was significantly higher compared with the WT mice (Figure 2A), reflecting enhanced whole-body insulin sensitivity. While neither the peripheral glucose uptake (Figure 2B) nor the EGP under the basal condition (Figure 2C) was affected, there was a significant reduction in EGP under 
A Body weight

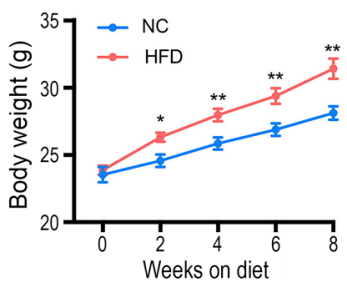

B Fasting glucose

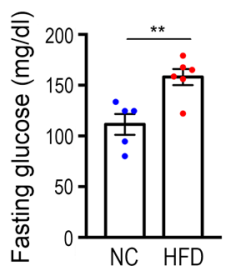

C GTT

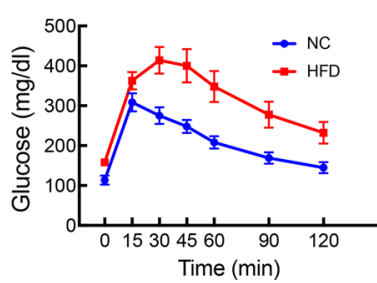

D Hepatic $\mathrm{H} 19$

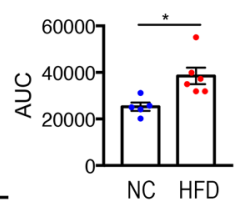

\section{E Hepatic $\mathrm{H} 19$}

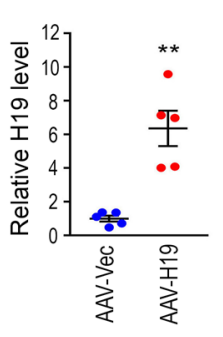

I GTT

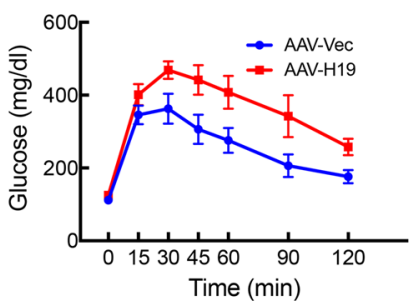

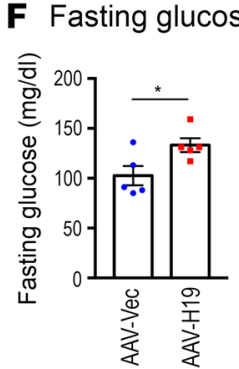

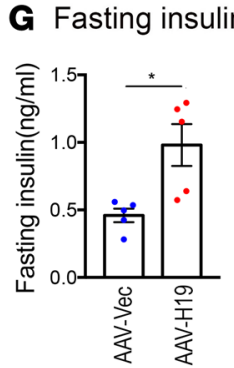

H PTT
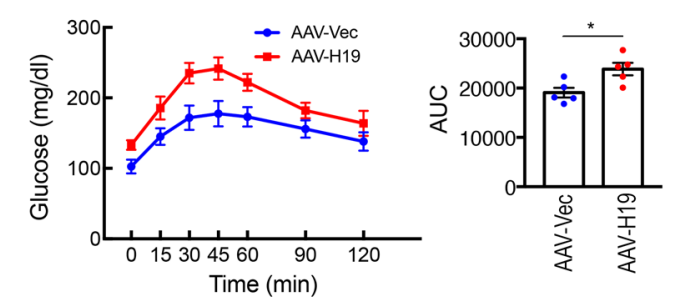

\section{J ITT}

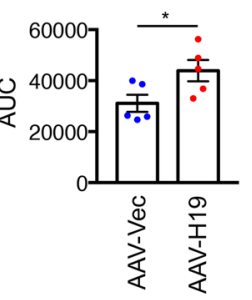

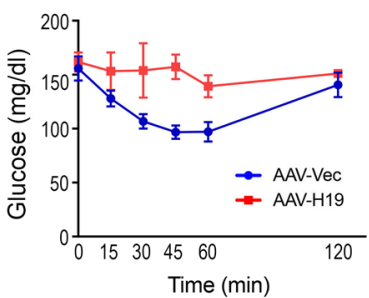

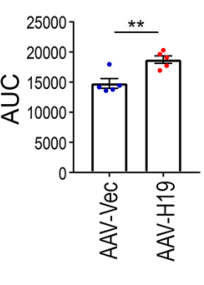

Figure 1. Effects of hepatic H19 upregulation on HGP and glucose homeostasis. (A) Body weight of mice fed with normal chow (NC) or high-fat diet (HFD). (B) Fasting plasma glucose levels. (C) Results of glucose-tolerance tests (GTTs). (D) Scatter plot of H19 levels in the liver. The horizontal line depicts group median, and the whiskers mark the interquartile range. (E) Scatter plot of $\mathrm{H} 19$ levels in livers of mice injected with AAV-Vec or AAV-H19. (F) Fasting blood glucose levels from mice injected with AAV-Vec or AAV-H19. (C) Fasting plasma insulin levels of mice injected with AAV-Vec or AAV-H19. (H) PTT results from mice injected with AAV-Vec or AAV-H19. (I) GTT results from mice injected with AAV-Vec or AAV-H19. (J) ITT results from mice injected AAV-Vec or AAV-H19. $n=5-6$ mice per group. Data are the mean \pm SEM. ${ }^{*} P<0.05$, ${ }^{* *} P<0.01$. Student $t$ test is used to compare differences between quantitative variables.

the clamp condition (Figure 2D) (see Discussion). Thus, the improved whole-body insulin sensitivity could be attributed to enhanced insulin-dependent suppression of EGP, which could be explained, at least in part, by the decreased expression of key gluconeogenic genes in the KO liver (Figure 2E). Taken together, these results suggest that $\mathrm{H} 19$ loss of function sensitizes the liver for insulin-dependent suppression of HGP.

H19 positively regulates gluconeogenic gene expression and glucose production. To provide mechanistic insights into the H19-dependent regulation of HGP observed in vivo, we used both HepG2 human hepatoma cells and primary mouse hepatocytes. H19 expression is robust in fetal hepatocytes but sharply declines in fully differentiated hepatocytes after birth. In human hepatoma cells, however, H19 expression is reactivated (19, 20). Thus, HepG2 cells were transfected with control siRNA (siCon) or H19-specific siRNA (siH19) (10), followed by glucose production assays. When $\mathrm{H} 19$ was downregulated (Figure 3A), the expression of Igf2 was not affected (Figure 3A), despite reports that $H 19$ and $I g f 2$ are coregulated in mouse skeletal muscle (8, 18). There was a significant decrease in glucose output in the H19-knockdown cells (Figure 3B), suggesting a positive role of $\mathrm{H} 19$ in glucose production. Next, we performed genome-wide transcriptome analysis in H19-knockdown HepG2 cells. RNA-seq analysis revealed a significant decrease in the expression of HNF4A, as well as key gluconeogenic enzyme genes $P C K 1$ and G6PC. These downregulations were also confirmed by quantitative PCR (qPCR) (Figure 3C) and Western blotting (Figure 3D). On the other hand, when H19 was overexpressed in primary mouse hepatocytes (known to express low levels of endogenous H19), there was an increase in expression of Hnf4a, Pck1, and G6pc at both the mRNA (Figure 3E) and protein (Figure 3F) levels, with a concomitant increase in glucose production (Figure 3G). Collectively, these findings indicate that $\mathrm{H} 19$ increases glucose production, at least in part by stimulating key gluconeogenic gene expression. 
A Glucose infusion rate

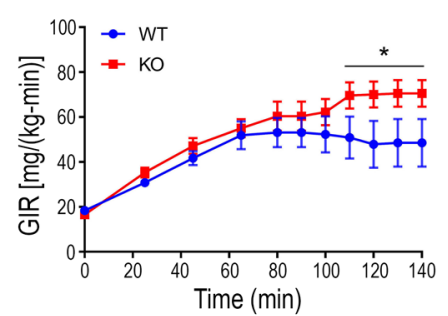

D Clamp EGP

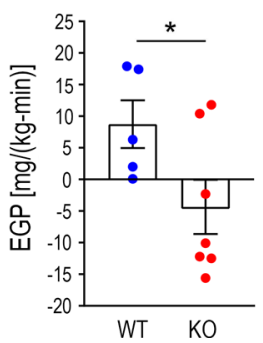

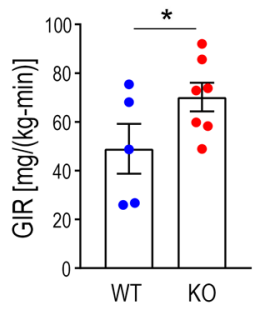

B Whole body uptake

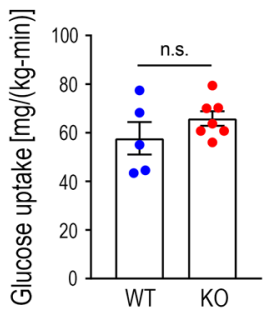

C Basal EGP

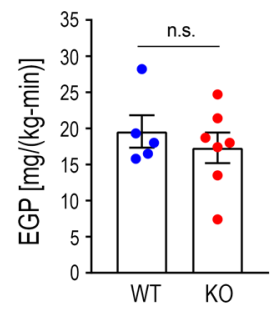

\section{E Western}
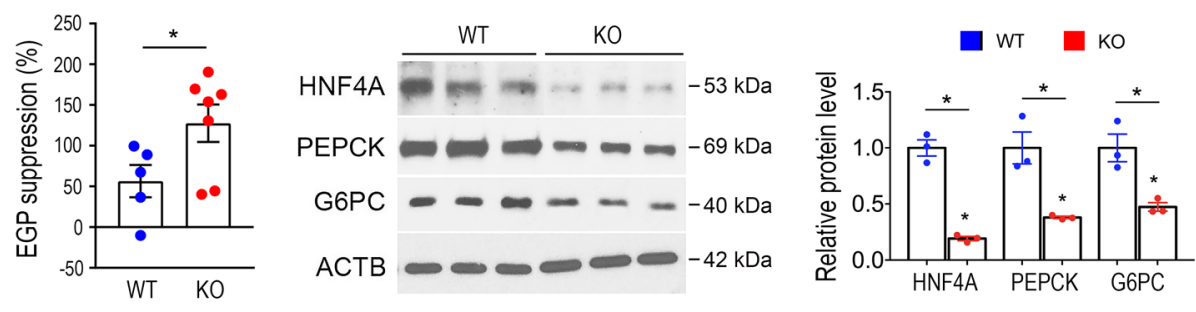

Figure 2. Hyperinsulinemic/euglycemic clamp studies of WT and KO mice. Compared with the WT mice, the KO mice showed an increased glucose infusion rate (A), unchanged whole-body glucose uptake (B), unchanged basal EGP (C), and increased insulin-stimulated EGP suppression (D). Western blots and corresponding densitometry show decreased gluconeogenic gene expression in KO versus WT mouse livers (E). $n=5-7$ mice per genotype. Quantification is based on 3 independent experiments. Numbers are the mean \pm SEM. ${ }^{*} P<0.05$ based on Student $t$ test.

$H 19$ regulates Hnf4a promoter methylation. In close inspection of our previous single nucleotide resolution genome-wide methylation data from human endometrial cancer cells (10) we noticed a correlation between H19 downregulation and increased methylation at multiple CpG sites within a 362-bp highly conserved promoter region of human $H N F 4 A$ (herein designated as differentially methylated region [DMR]) (Figure 4A and Supplemental Figure 2). To determine whether H19 knockdown in HepG2 cells might also lead to increased methylation of the DMR, quantitative methylation-specific PCR (QMSP) was performed using previously described methods $(9,10)$. The QMSP primers were designed based on the differentially methylated cytosine residues within the DMR (Supplemental Figure 2). As shown in Figure 4B, cells treated with siH19 had an increase in promoter methylation compared with cells treated with siCon. As increased promoter methylation often represses gene expression, there was indeed a decrease in the Hnf $4 \mathrm{a}$ mRA level in siH19- versus siCon-treated cells (Figure 4C).

As H19 is known to bind SAHH and inactivate it, leading to decreased DNA methylation $(9,10)$, we tested whether this $\mathrm{H} 19 / \mathrm{SAHH}$ pathway might be involved in the regulation of $H N F 4 A$ methylation. As expected, in the presence of D-Eritadenine (DEA), a SAHH-specific inhibitor that mimics the inhibitory function of H19, both methylation (Figure 4B) and mRNA (Figure 4C) of HNF4A were restored to control levels. To provide further evidence for the involvement of the H19/SAHH pathway, RNA IP (RIP) experiments using a monoclonal antibody specific to SAHH (9) with AAV-H19-infected mouse livers were carried out. A nearly 8-fold enrichment of H19 in SAHH-containing RNA-protein complexes relative to control IgG immunoprecipitates was observed, whereas Gapdh mRNA, expressed at a 100-fold higher level than that of H19, was not detected in the SAHH-containing complexes (Figure 4D), indicating a physical interaction between $\mathrm{H} 19$ and SAHH in hepatocytes. Taken together, these results suggest that $\mathrm{H} 19$ regulates promoter methylation and expression of Hnf4a in hepatocytes and that this regulation is likely dependent on the H19/SAHH pathway.

Fasting temporally upregulates hepatic $H 19$ and promotes hypomethylation and expression of Hnf4a. To test whether the H19-mediated regulation of Hnf4a promoter methylation and expression occurs in vivo, we took advantage of the fasting mouse model, which is known to increase HGP. Thus, WT mice were subjected to 12-hour overnight fasting, followed by gene expression and methylation analysis. Overnight fasting resulted in upregulation of $\mathrm{H} 19$, together with $H n f 4 a, P c k 1$, and G6pc at levels of both mRNA (Figure 5A) and protein (Figure 5B). Thus, H19 expression is temporally induced by fasting under physiological conditions, which was not previously documented in the literature. During fasting, glucagon is the main peptide hormone secreted from the $\alpha$ cells of the pancreas. It acts on the glucagon receptor 

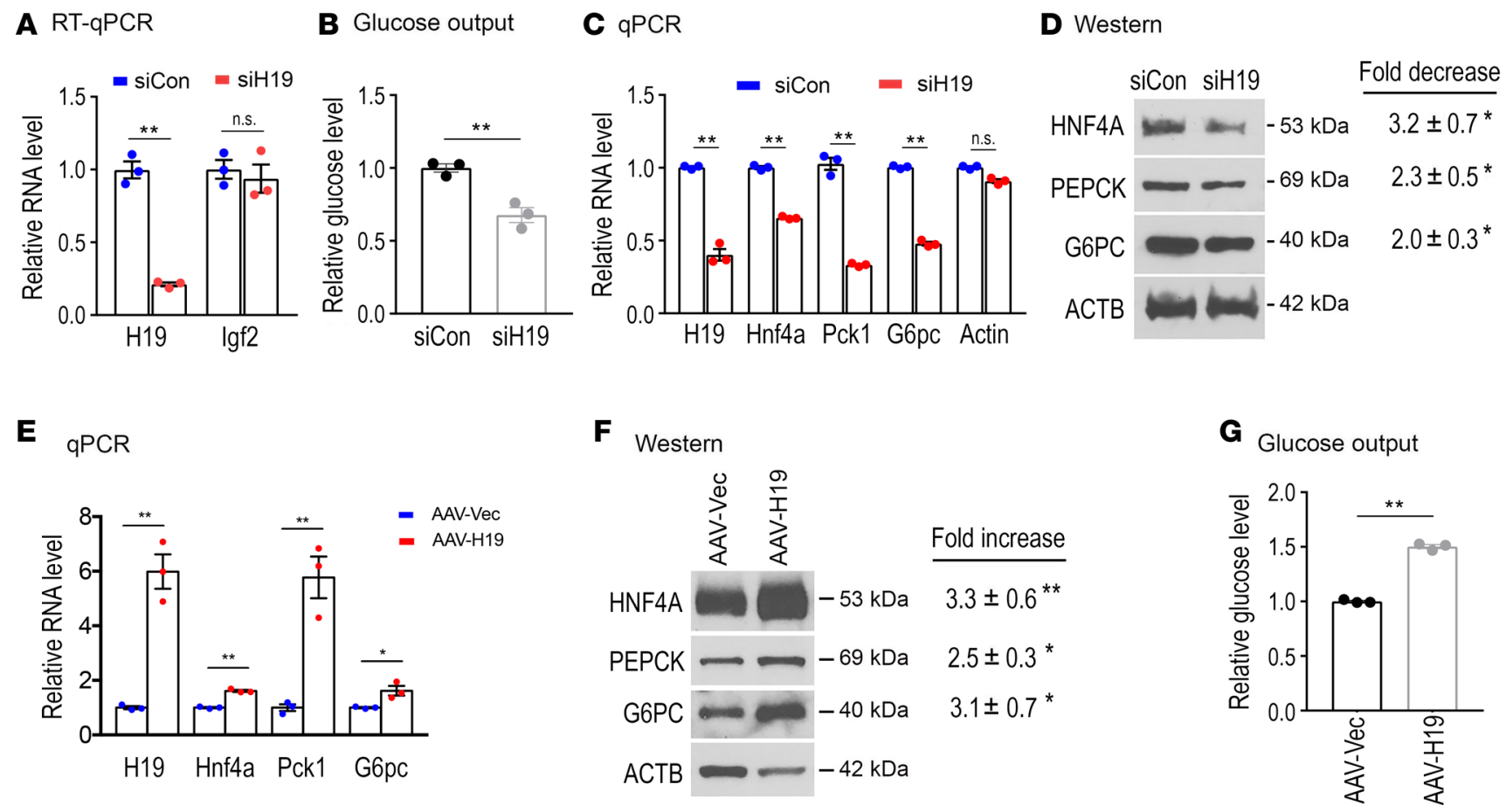

Figure 3. Effects of $\mathbf{H 1 9}$ expression on gluconeogenic gene expression and glucose output in vitro. (A) HepG2 cells were transfected with siCon or siH19, followed by RNA extraction and qPCR analysis 48 hours later. Relative RNA levels are presented. (B) HepG2 cells were transfected with siCon or siH19, and glucose output assays were performed 48 hours later. Relative glucose outputs are presented. (C) qPCR results of HepG2 cells transfected with siCon or siH19 at 48-hour time point. (D) HepG2 cells were transfected with siCon or siH19, followed by Western blot analysis 48 hours later. Representative gel images from 3 transfection experiments are shown, with fold decreases in siH19 compared with siCon transfected cells marked on the right. (E-C) Mouse primary hepatocytes were infected with AAV-Vec or AAV-H19 viruses, followed by qPCR and Western blot analyses at 48 hours and glucose output assays at 72 hours. Quantification is based on 3 independent transfection/infection experiments. Numbers are the mean $\pm \mathrm{SEM}$. ${ }^{*} P<0.05$, ${ }^{* *} P<0.01$ based on Student $t$ test.

to increase cAMP, which activates cAMP-dependent protein kinase A (PKA), leading to enhanced gluconeogenic gene expression (1). Incubation of HepG2 cells with glucagon stimulated H19 expression, an effect that was mimicked by cAMP and abolished by H89, a pharmacological inhibitor of PKA (21) (Figure $5 \mathrm{C}$ ). These results suggest that glucagon released during fasting may contribute to H19 upregulation and that the CAMP-PKA signaling may be involved in this regulation.

As decreased H19 expression increases promoter methylation of HNF4A in cultured human cells (Figure 4B), we predicted that fasting (which increases H19 expression) would decrease Hnf4a promoter methylation and subsequently increase its expression. Indeed, our genome-wide single-nucleotide resolution DNA methylation analysis revealed decreased methylation at multiple CpG sites within the conserved promoter region of Hnf4a in fasted versus control mouse livers (Figure 4A and Supplemental Figure 3A), which was further confirmed by QMSP (Figure 5D). In sharp contrast to Hnf4a, whose promoter became hypomethylated in response to fasting, the promoters of both G6pc and Pck1 showed hypermethylation (Supplemental Figure 3, B and C). This suggests that the increase in expression of G6pc and Pck1 in fasted liver is likely not a direct effect of $\mathrm{H} 19$ but rather a result of HNF4A-dependent regulation. Collectively, these results suggest that acute H19 upregulation induces Hnf4a promoter hypomethylation and expression, contributing to the subsequent increase in key gluconeogenic gene expression and HGP under physiological conditions.

Chronic alteration of $\mathrm{H} 19$ expression affects Hnf4a methylation and gluconeogenic gene expression. We have induced hepatic H19 upregulation by HFD feeding (Figure 1D) or by AAV-H19 injection (Figure 1E), and we have used the H19-KO mouse model to test the effects of H19 depletion. Remarkably, when H19 was chronically increased by HFD feeding, there was a decrease in Hnf4a promoter methylation (Figure 6A) with a concomitant increase in Hnf4a mRNA (Figure 6B). As expected, the expression of the key gluconeogenic genes was also increased (Figure 6C). A similar situation was observed when H19 was overexpressed by AAV-H19 injection (Figure 6, D-F). On the other hand, when H19 was depleted, there was an increase in Hnf4a promoter methylation (Figure 6G), a decrease in Hnf4a mRNA (Figure 6H), and a decrease in expression of key gluconeogenic genes (Figure 6I). Thus, there is a strong in vivo positive 


\title{
A DMR sequences
}

\author{
Human chr20 42984045 \\ $\downarrow$ \\ GAGCCATAAAACCCAACCCAGGTTGGACTCTCACCTCTCCAGCCCCTTCTGCTCCGGCCCTGTCCTCAAATTGGGGGGCTGATGTCCCCATA
}

CACCTGGCTCTGGGTTCCCCTAACCCCAGAGTGCAGGACTAGGACCCGAGTGGACCTCAGGTCTGGCCAGGTCGCCATTGCCATGGAGACAGCA

ACAGTCCCCAGCCGCGGGTTCCCTAAGTGACTGGTTACTCTTTAACGTATCCACCCACCTTGGGTGATTAGAAGAATCAATAAGATAACCGGGCGG

Mouse chr2 163506534

$\downarrow$

GAGCCATAAAACTCCACCCATCACGGACTCTCAACAATTCCCTAGGCCCTGCTGCTCAGCCTCCTCTCTCAAGTTGGAAGGCTGATGTTTC

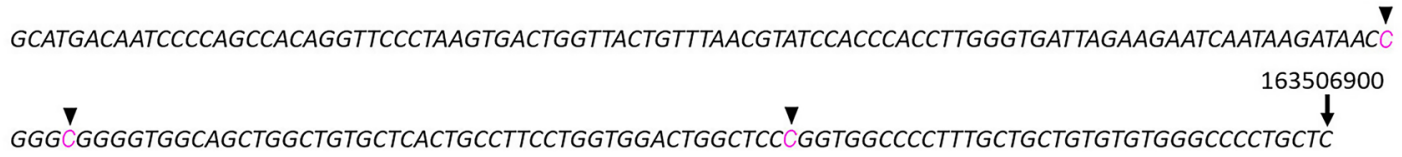

B QMSP

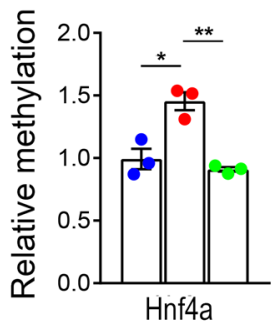

siCon c $\mathrm{qPCR}$

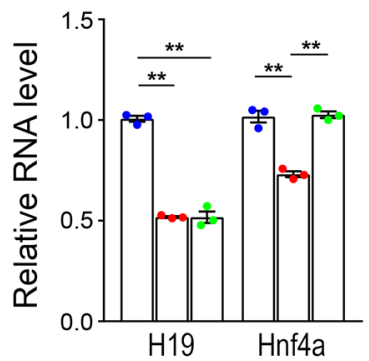

$\mathrm{siH} 19$

siH19+DEA
D RIP + qPCR
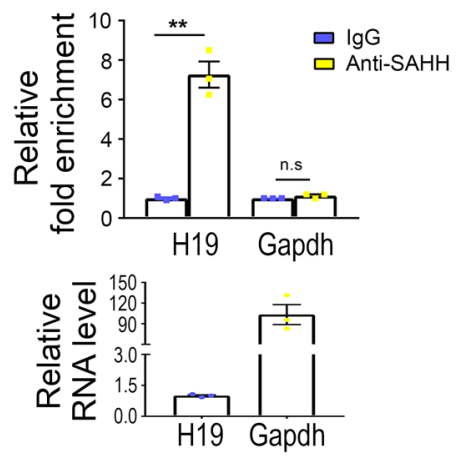

Figure 4. Regulation of HNF4A promoter methylation via the H19/SAHH pathway. (A) Sequences of DMRs in the conserved promoter region of human and mouse Hnf4a. The 3 differentially methylated cytosine residues are indicated. The numbers on top of the sequences mark the positions of the indicated nucleotides in the chromosomes. The 3 differentially methylated cytosine residues are indicated in pink with arrowheads. (B) HepC2 cells were transfected with siCon, siH19, or siH19 + DEA. Genomic DNAs were extracted 15 hours later and analyzed by QMSP. (C) HepG2 cells were treated as described in B. RNAs were extracted 24 hours later and analyzed by qPCR. (D) RIP with anti-SAHH or preimmune IgGs from extracts of mouse livers. Top panel: RNA levels in immunoprecipitates were determined by qPCR. Levels of H19 and Gapdh mRNA are presented as fold enrichment in anti-SAHH relative to IgG immunoprecipitates. Bottom panel: relative RNA levels of H19 and Gapdh in mouse livers. Quantification is based on 3 independent experiments. Data are the mean \pm SEM. ${ }^{*} P<0.05,{ }^{* *} P<0.01$ based on Student $t$ test.

correlation between $\mathrm{H} 19$ expression, Hnf4a promoter hypomethylation, Hnf4a mRNA level, and gluconeogenic protein levels in all cases tested so far (Figure 6, A-I, and Figure 5, A, B, and D).

Insulin suppresses hepatic gluconeogenesis by binding to and initiating signaling through the insulin receptor (INSR). One critical downstream pathway is the insulin-stimulated PI3K-mediated phosphorylation of AKT at Ser473, leading to direct inhibition of transcription of Pck1 and G6pc (reviewed in ref. 2). To rule out the possibility that the H19-induced increase in expression of $P c k 1$ and G6pc (Figure 6F) might be a result of decreased insulin signaling, INSR expression and AKT phosphorylation at Ser473 were analyzed. An increase in both INSR expression (Figure 6J) and AKT phosphorylation (Figure 6J) in AAV-H19 as compared with AAV-Vec-infected livers was observed, suggesting enhanced insulin signaling in the H19-overexpressed liver. While the increased insulin signaling could be due to a compensatory 
A $\mathrm{qPCR}$

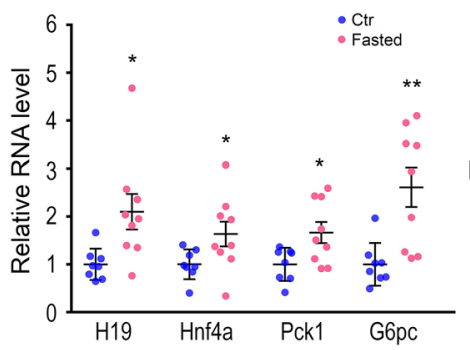

B Western

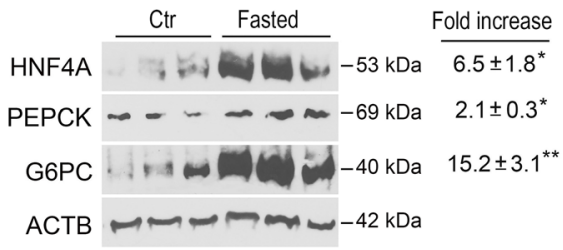

C HepG2

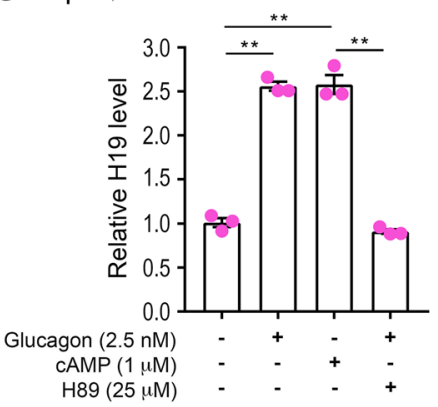

D QMSP

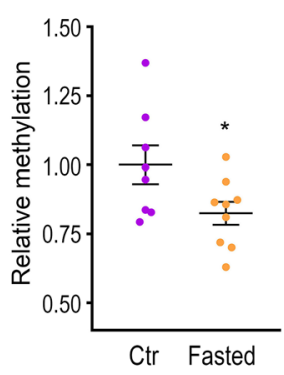

Figure 5. Effects of fasting on H19 and gluconeogenic gene expression. (A) WT mice were fasted overnight. qPCR analysis was performed on liver samples collected from fasted and nonfasted (Ctr) animals. Scatter plot of RNA levels is shown. (B) WT mice were overnight fasted, followed by protein analysis of the indicated genes by Western blotting. (C) HepG2 cells were treated with the indicated reagents for 2 hours. Relative H19 levels are presented. (D) Scatter plot of Hnf4a methylation as assessed by QMSP in fasted and control livers. $n=8-9$ animals. Quantification is based on 3 independent experiments. Data are the mean \pm SEM. ${ }^{*} P<0.05,{ }^{* *} P<0.01$ based on Student $t$ test.

mechanism, these results further support the notion that the H19/Hnf4a pathway likely constitutes an important mechanism of hepatic gluconeogenic gene activation.

Igf2 and miR-675 are not involved in H19-mediated regulation of HGP. To test whether miR-675 encoded by H19 (22) and/or Igf2 (which is coregulated with H19 in skeletal muscle; ref. 18) contribute to the H19-mediated regulation of HGP, hepatic expressions of miR-675 and Igf2 were evaluated. In WT mice, no significant difference in levels of Igf2 mRNA (Supplemental Figure 4A) and miR-675 (Supplemental Figure 4B) was detected between control and fasted livers, nor was there a significant difference in Igf2 expression between WT and KO livers (Supplemental Figure 4C). These results are consistent with previous studies reporting lack of coregulation of H19 with miR-675 and $\operatorname{Ig} 2$ in livers $(11,23)$. Taken together with the notion that Igf2 expression was not affected in H19-knockdown HepG2 cells (Figure 3A), we conclude that Igf2 and miR-675 are likely not contributing to the H19-dependent regulation of HGP.

\section{Discussion}

We have found that H19, which is normally expressed at a low but appreciable level in the liver, is temporally increased by fasting under physiological conditions. This acute H19 elevation leads to decreased promoter methylation and increased expression of Hnf4a and subsequent activation of the gluconeogenic program. Importantly, this lncRNA-mediated mechanism is shared by pathological conditions where a chronically elevated H19 is seen both in animals (Figure 1D) and in human subjects with T2D (12). A proposed model is shown in Figure 6K.

While acute insulin-stimulated repression of gluconeogenesis can occur without alteration in gluconeogenic gene expression (24), in chronic diabetic states, inhibition of rate-limiting gluconeogenic enzyme production has been shown to reduce HGP and improve insulin sensitivity (25). Along the same lines, in the H19-KO liver, the enhanced insulin-mediated suppression of HGP (Figure 2D) can be ascribed, at least in part, to decreased expression of the rate-limiting enzymes (Figure 2E). On the other hand, liver-specific H19 overexpression in WT mice leads to hypomethylation of Hnf4a (Figure 6D) and increased gluconeogenic enzyme production (Figure 6, E and F) and HGP (Figure 1H). It is thus conceivable that, under diabetic conditions, the chronic increase in hepatic H19 contributes to elevated levels of gluconeogenic enzymes, thereby reducing the ability of insulin to suppress HGP.

The lack of reduction in EGP under the basal condition in the KO versus WT livers (Figure 2C) was initially unexpected. Under normal conditions, the liver is the major source of EGP during fasting. However, mice with liver-specific deletion of G6pc (therefore incapable of HGP) were able to maintain normal fasting plasma glucose levels due to compensatory induction of gluconeogenesis in the kidney and intestine $(26,27)$. Thus, a compensatory induction of extrahepatic gluconeogenesis in the H19-KO mice during fasting might have helped to maintain the basal EGP.

We have previously reported that H19 downregulation by siRNA in mouse myotubes or by metformin treatment in human endometrial cancer cells promotes extensive global DNA methylation changes and that the interaction between $\mathrm{H} 19$ and SAHH constitutes 1 underlying mechanism (the H19/SAHH regulatory axis) 

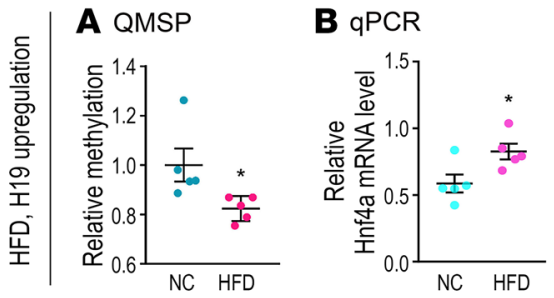

C Western
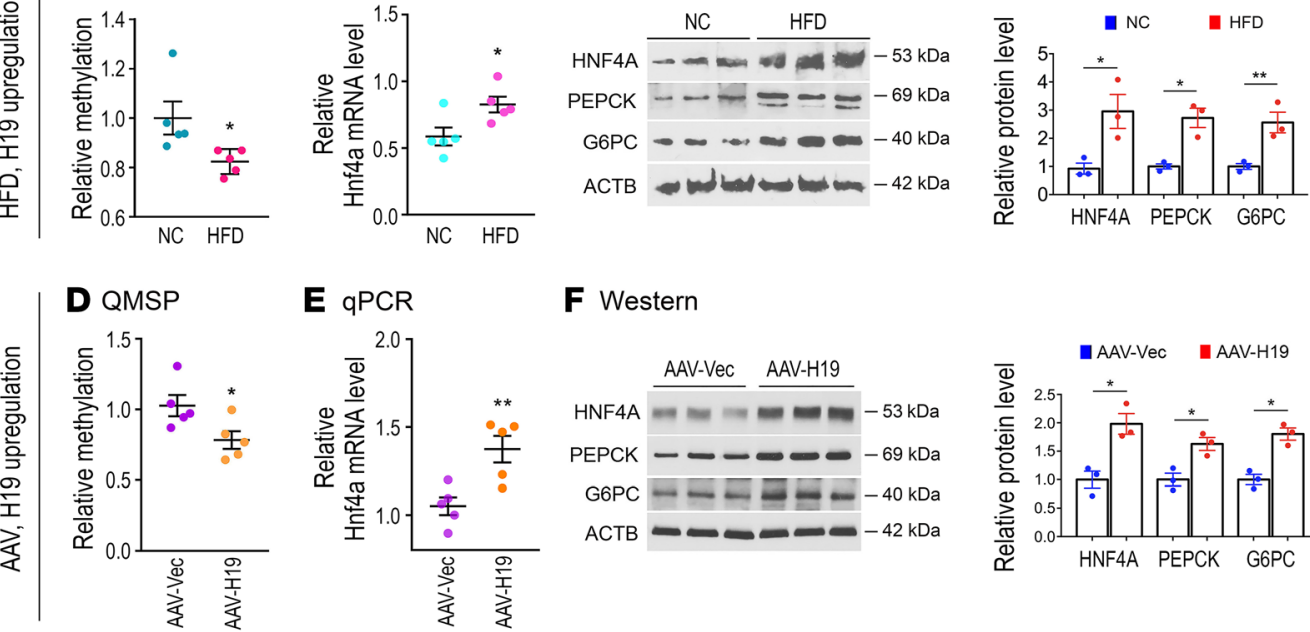

E qPCR

F Western
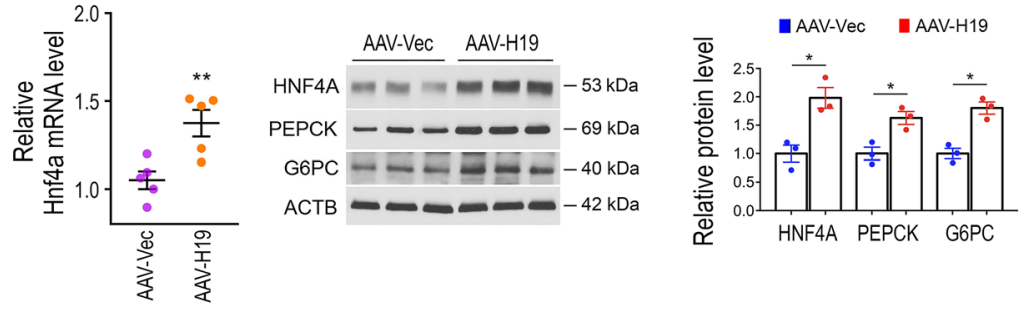

Western

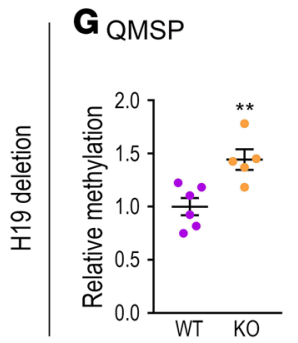

$\mathbf{H}_{\mathrm{qPCR}}$

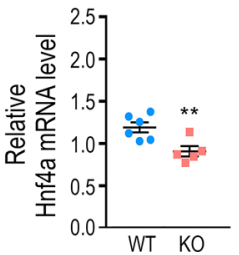

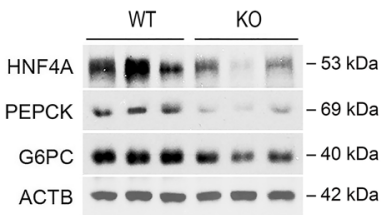

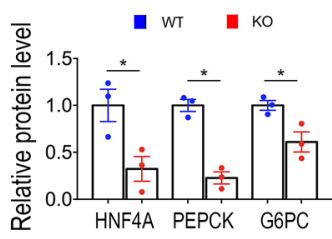

J Western

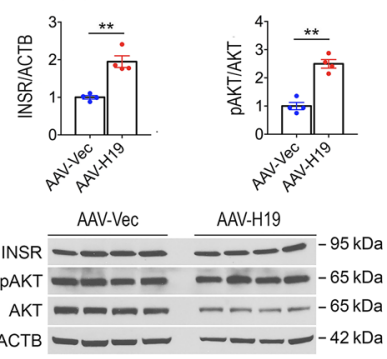

K Mode

\section{Fasting//2D}

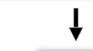

4 HI9

$\perp$

$\downarrow$ Hnf4a methylation

$\perp$

१ Hnf4a expression

$\downarrow$

$\uparrow$ Gluconeogenic gene expression

$\downarrow$

Gluconeogenesis

I

Enhanced glucose production

Figure 6. Effects of $\mathrm{H} 19$ expression on hepatic Hnf4a promoter methylation, gluconeogenic gene expression, and insulin signaling in vivo. Genomic DNAs were extracted from livers of NC and HFD mice (A), AAV-Vec and AAV-H19 mice (D), and WT and KO mice (C) fed ad libitum. Hnf4a promoter methylation was assessed by QMSP. (B, E, H) Relative Hnf4a mRNA levels as assessed by qPCR. (C, F, I) Relative protein levels as assessed by Western blotting. (J) Effects of H19 overexpression on hepatic insulin signaling. Proteins were extracted from livers of AAV-Vec- or AAV-H19-infected mice fed ad libitum and subjected to Western blot analysis. Quantifications (top panels) of INSR protein levels and AKT phosphorylation at Ser473 of the Western blots (bottom panel) are shown. $n=4-5$ animals per group. Numbers are the mean \pm SEM. ${ }^{*} P<0.05,{ }^{*} P<0.01$ based on Student $t$ test. (K) A proposed model.

$(9,10)$. While the mechanism dictating H19/SAHH-mediated target gene selection remains to be investigated, we were able to predict and subsequently confirm (by both QMSP and genome-wide DNA methylation profiling) the direction (increase or decrease) and sites of Hnf4a methylation in both human (HepG2) and mouse (liver). This strongly suggests that the highly conserved promoter region of Hnf4a is likely a direct target of the H19/SAHH-mediated regulation. The causative role of H19 in inducing Hnf4a promoter hypomethylation is further supported by both our in vitro (HepG2) and in vivo (liver-specific H19 overexpression and wholebody H19-KO mouse models) studies, as well as by an independent study demonstrating a positive correlation between H19, HNF4A promoter hypomethylation, and expression in human gastric cancer (28). It is important to note that the H19-induced changes in the levels of Hnf4a promoter methylation were modest, while stronger changes in its protein levels were observed. This suggests that other mechanisms are involved in the regulation of Hnf4a expression. Likewise, HNF4A-independent pathways are most likely also involved in the H19-mediated regulation of gluconeogenesis.

A recent study reported decreased $\mathrm{H} 19$ expression in the liver of diabetic $\mathrm{db} / \mathrm{db}$ mice and that siRNA-mediated H19 knockdown in HepG2 and primary mouse hepatocytes resulted in increased gluconeogenic gene expression and glucose production (29). These results are opposite to ours reported here. While the reason for this apparent discrepancy is unclear, it is noteworthy to point out that our results have been derived from multiple animal models (both H19 loss of function and gain of function, HFD, and overnight fasting) and are consistent with human studies reporting increased hepatic H19 expression in diabetic patients (12).

It has been well established that gluconeogenesis is activated by glucagon and inhibited by insulin. The upregulation of H19 by glucagon in hepatic cells (Figure 5C) further supports a role of H19 in gluconeogenesis activation. The glucagon-stimulated H19 upregulation appears to be via the cAMP/PKA pathway, which is interesting because H19 upregulation by cAMP analogs or PKA activators has been 
reported to occur in primary cultures of human fetal adrenal cells, as well as adult human adrenocortical cells $(30,31)$. While glucagon may promote hepatic H19 expression during fasting in healthy individuals, it may also contribute to the chronic increase in H19 in liver of T2D patients (12). It has long been known that T2D patients have chronic hyperglucagonemia, in part due to inappropriately increased $\alpha$ cell function $(32,33)$. The detailed molecular mechanism by which glucagon regulates hepatic H19 expression remains to be determined.

In summary, the findings presented in this study represent the first example to our knowledge of a lncRNA-mediated mechanism in the pathogenesis of diabetic hyperglycemia and suggest that targeting hepatic H19 may be a new option for the treatment of this disease.

\section{Methods}

Antibodies and reagents. Antibodies for G6PC (ab83690 [for human G6PC], used at a dilution of 1/500, from Abcam; LS-C446262 [for mouse G6PC], used at dilution of 1:1,000, from LifeSpan BioSciences), HNF4A (Abcam, ab181604; used at a dilution of 1/1,000), PEPCK (Abcam, ab70358; used at a dilution of 1/1,000), INSR- $\beta$ (A303-712A, Bethyl Laboratories Inc., used at a dilution of 1/1,000), pAKT Ser473 (9271L, Cell Signaling Technology, used at a dilution of 1/1,000), AKT (9272S, Cell Signaling Technology, used at a dilution of 1/1,000), and $\beta$-actin (Cell Signaling Technology, 4967; used at a dilution of 1/5,000) were purchased. Human H19 siRNA (siH19) and siCon were previously described (34). Glucagon (G20441MG, MilliporeSigma) and DEA (sc-207632, Santa Cruz Biotechnology Inc.) were purchased. DEA was used at a final concentration of $20 \mu \mathrm{M}$.

Animals. All mice used in this report were male. CD-1 mice were obtained from Charles River Laboratories. The H19-KO mice on the background of C57BL/6J were gifts from Luisa Dandolo (Institut Cochin, Paris, France). Mice were housed at $22^{\circ} \mathrm{C}-24^{\circ} \mathrm{C}$ with a 12-hour light/12-hour dark cycle with standard chow (Purina Chow; Purina Mills) and water provided ad libitum. For fasting experiments (Figure 5, A, B, and D), 12-week-old CD-1 mice were fasted for 12 hours and then sacrificed by ether anesthesia followed by cervical dislocation. Liver samples were collected and snap frozen in liquid nitrogen and stored at $-80^{\circ} \mathrm{C}$ for further analysis. For HFD experiments (Figure 1, A-D), WT C57BL/6J mice at 9 weeks old were exposed to HFD for 8 weeks, followed by the indicated tests.

$A A V$ production and in vivo AAV administration. The AAV-H19 expression plasmid was created by cloning a mouse H19 full-length DNA (NR_001592) into an AAV8-TBG vector (Vigene Biosciences). Viruses were amplified, packaged, and purified by Vigene. WT CD-1 mice at 16 weeks old were tail-vein injected with AAV-H19 or AAV-Vec (as negative control) at $1 \times 10^{10} \mathrm{gc} /$ mouse in $150 \mu \mathrm{of} \mathrm{PBS} / 5 \%$ sorbitol (Vigene Biosciences). The viral dose was based on a previously published report (14). Before injection, mice were exposed to heat lamp to dilate the tail vein and then placed in a restrainer permitting access to the tail vein. The tail was cleansed with 70\% ethanol (Decon Labs), and the injection was made in the lateral vein, using 27-gauge needles.

PTT, glucose-tolerance test (GTT), and insulin-tolerant test (ITT). PTT, GTT, and ITT were carried out as previously described (35). Briefly, mice injected with AAV-H19 or AAV-Vec were subjected to PTT at 2 weeks following the viral injection. PTT was performed in 16-hour-fasted animals. Mice received an i.p. injection of $2 \mathrm{~g} / \mathrm{kg}$ body weight sodium pyruvate (MilliporeSigma, P5280). Blood glucose concentrations were measured from blood samples obtained from the tail vein at 15, 30, 45, 60, 90, and 120 minutes after the injection. Fasting plasma insulin levels were measured using Mouse Insulin ELISA kit (MilliporeSigma, EZRMI-13K) according to the manufacturer's instructions. For GTT, following overnight (12-hour) fasting, the basal level of blood glucose was measured. Glucose (1 g/kg; MilliporeSigma, G5767) was injected i.p., and the blood glucose concentration was measured from a blood sample obtained from the tail vein 15, 30, 45, 60, 90, and 120 minutes after the glucose injection. ITTs were performed in ad libitum-fed mice. Each animal received an i.p. injection of insulin at $1 \mathrm{U} / \mathrm{kg}$ (Humulin R; Eli Lilly). Blood glucose levels were monitored at 0, 15, 30, 45, 60, and 120 min after the injection.

Hyperinsulinemic-euglycemic clamp studies. The experiments were carried out on 11-week-old WT and H19KO mice as previously described with minor modification (36) (Figure 2, A-D). Briefly, mice were anesthetized and cannulated. After recovery for 7 days, mice were fasted overnight (14 hours), followed by infusion of $\mathrm{D}-\left[3-{ }^{3} \mathrm{H}\right]$ glucose to assess the basal rate of whole body glucose turnover. Following the basal period, a 2-hour hyperinsulinemic-euglycemic clamp was conducted with a fixed amount of insulin (4 mU/[kg-min]) and a variable amount of $20 \%$ dextrose to maintain euglycemia. Tissues were collected at the end of the clamp. 
Cell culture, transfection, and AAV infection. Mouse primary hepatocytes were prepared as previously described (37). For H19-overexpression experiments, freshly isolated mouse primary hepatocytes were seeded in 12-well plates at $4 \times 10^{5}$ cells/well and infected with AAV-Vec or AAV-H19 at 2,000 gc/cell at 2 hours after seeding. RNA and proteins were extracted at 48 hours after infection for qPCR and Western blot analyses. Glucose output assays were carried out at 72 hours after infection. HepG2 cells (American Type Culture Collection, ATCC HB-8065) were authenticated and were free from mycoplasma contamination. The cells were maintained in ATCC-formulated Eagle's Minimum Essential Medium (EMEM; ATCC30-2003) supplemented with 10\% FBS, heat inactivated FBS, and 1\% penicillin/streptomycin. Cells were transfected in a 24-well plate scale. To prepare siRNA transfection solution for each well, 2 pmol of siCon or siH19 was mixed with $100 \mu \mathrm{l}$ OPTI-MEM (Gibco) by gentle pipetting. In parallel, $1 \mu 1$ Lipofectamine 2000 (Thermo Fisher Scientific) was mixed with $100 \mu 1$ OPTI-MEM. Following 5 minutes of incubation at room temperature, the 2 were mixed by gentle pipetting and incubated for 20-30 minutes at room temperature to allow siRNA/lipid complexes to form. At the end of incubation, the $200-\mu 1$ transfection solution was used to resuspend cell pellet $\left(2 \times 10^{5}\right.$ cells). After incubation at room temperature for 10 minutes, regular growth medium was added at a ratio of 1:2 (1 volume of transfection solution/2 volumes of growth medium), and the cell suspension was transferred to the culture plate. After 5-hour incubation at $37^{\circ} \mathrm{C}$ in $5 \% \mathrm{CO}_{2}$, the medium was replaced with fresh growth medium. RNA, genomic DNA, and protein were extracted and analyzed at the indicated time points following transfection.

Methyl-MiniSeq library construction. Libraries were prepared from 200-500 ng of genomic DNA digested with 60 units of Taq $\mathrm{I}$ and 30 units of MspI (NEB) sequentially and then extracted with Zymo Research (ZR) DNA Clean \& Concentrator-5 kit (catalog D4003). Fragments were ligated to preannealed adapters containing 5-Methylcytosine instead of cytosine according to Illumina's specified guidelines (www.illumina.com). Adaptor-ligated fragments of 150-250 bp and 250-350 bp in size were recovered from a $2.5 \%$ NuSieve 1:1 agarose gel (Zymoclean Gel DNA Recovery Kit, ZR, D4001). The fragments were then bisulfite-treated using the EZ DNA Methylation-Lightning Kit (ZR, D5020). Preparative-scale PCR was performed, and the resulting products were purified (DNA Clean \& Concentrator, ZR, D4005) for sequencing on an Illumina HiSeq.

Methyl-MiniSeq sequence alignments and data analysis. Sequence reads from bisulfite-treated EpiQuest libraries were identified using standard Illumina base-calling software and then analyzed using a ZR proprietary analysis pipeline, which is written in Python and used Bismark (http://www.bioinformatics. babraham.ac.uk/projects/bismark/) to perform the alignment. Index files were constructed using the bismark_genome_preparation command and the entire reference genome. The non_directional parameter was applied while running Bismark. All other parameters were set to default. Filled-in nucleotides were trimmed off when doing methylation calling. The methylation level of each sampled cytosine was estimated as the number of reads reporting a $C$, divided by the total number of reads reporting a $C$ or T. Fisher's exact test or 2-tailed Student's $t$ test was performed for each CpG site that has at least 5 reads coverage, and promoter, gene body, and $\mathrm{CpG}$ island annotations were added for each $\mathrm{CpG}$ included in the comparison. Methylation data are available at GEO accession GSE103437 (https://www.ncbi.nlm. nih.gov/geo/query/acc.cgi?acc=GSE103437).

RNA-seq and data analysis. HepG2 cells were transfected with siCon or siH19 in a 6-well plate. Cells were harvested for RNA extraction 48 hours after transfection using the Purelink RNA mini kit (Ambion, 12183018A). RNA-seq libraries were prepared using the Illumina TruSeq Stranded Total RNA LT kit with Ribo-Zero Human/Mouse/Rat, setA (rs-122-2201) according to the sample preparation protocol. Briefly, $1 \mu \mathrm{g}$ of total RNA was subjected to Ribo-Zero depletion to remove ribosomal RNAs (rRNAs). The remaining RNA was purified, fragmented, and primed with random hexamers for cDNA synthesis. After first and second cDNA synthesis, cDNA fragments were adenylated and then ligated to indexing adapters. The cDNA fragments were enriched by PCR, purified, and then sequenced on an Illumina NextSeq500 using paired-end chemistry and 76-bp cycles. Sequences are available from the GEO with accession number of GSE103437. Illumina BaseSpace-embedding tools (https://basespace.illumina.com/) were used to analyze the RNA-seq data. RNA-Seq Alignment v1.0.0 was used to map sequencing reads to mm10 genome and quantify reads of genes. DESeq2 v1.0.0 was applied to calculate differential expression of genes.

$R N A$ extraction and $q P C R$. Total RNAs were extracted from liver tissue samples or from HepG2 cells using PureLink RNA Mini Kit (Ambion, 12183018A). cDNA was synthesized using PrimeScript RT Reagent Kit (TAKARA, RR037A) in a $20-\mu 1$ reaction containing $0.5-1 \mu \mathrm{g}$ of total RNA. qPCR was 
performed in a 15- $\mu 1$ reaction containing $0.5-1 \mu 1$ of cDNA using iQSYBRGreen (Bio-Rad) in a Bio-Rad iCycler. PCR was performed by initial denaturation at $95^{\circ} \mathrm{C}$ for 5 minutes, followed by 40 cycles of 30 seconds at $95^{\circ} \mathrm{C}, 30$ seconds at $60^{\circ} \mathrm{C}$, and 30 seconds at $72^{\circ} \mathrm{C}$. Specificity was verified by melting curve analysis and agarose gel electrophoresis. The Ct values of each sample were used in the post-PCR data analysis. Gene expression levels were normalized against housekeeping genes Hprt1 and Rplp0. Real-time PCR primers are listed in Supplemental Table 1.

Western blot analysis. Cells in 24- or 12-well plates were quickly lysed in situ in $2 \times$ SDS-sample buffer $\left(100 / 200 \mu 1 /\right.$ well), followed by heating at $100^{\circ} \mathrm{C}$ for 5 minutes, with occasional vortexing. In the case of liver samples, $\sim 10 \mathrm{mg}$ of frozen tissues were homogenized in $200 \mu 1$ of $2 \times$ SDS-sample buffer and then heated at $100^{\circ} \mathrm{C}$ for 5 minutes, with occasional vortexing. Homogenized samples were loaded onto $12 \%$ SDS gel ( $5 \mu \mathrm{g} /$ well; Bio-Rad), followed by Western blot analysis. The linear dynamic range of each protein of interest was determined by serial dilutions. Bands on Western blot gels were quantified using ImageJ. $\beta$-Actin was used as a loading control.

$R I P$. To prepare antibodies, $10 \mu 1$ of protein G Sepharose beads (Santa Cruz Biotechnology Inc., sc-2002) were incubated with $20 \mu \mathrm{g}$ of monoclonal anti-SAHH antibody (Santa Cruz Biotechnology Inc., sc-271389) or $20 \mu \mathrm{g}$ of mouse preimmune IgG (Chemicon, PP54) in $500 \mu 1$ of IP buffer $(0.5 \%$ Triton X-100, $200 \mathrm{mM} \mathrm{NaCl}, 10 \mathrm{mM}$ Tris- $\mathrm{HCl}$ at $\mathrm{pH}$ 7.5, and $10 \mathrm{mM}$ EDTA) at $4^{\circ} \mathrm{C}$ overnight. The next day, the beads were washed $3 \times$ with IP buffer and kept on ice until used. To prepare tissue lysates, $30 \mathrm{mg}$ of frozen liver tissue was manually homogenized on ice in $900 \mu \mathrm{l}$ of freshly prepared lysis buffer (0.5\% Triton X-100, $10 \mathrm{mM} \mathrm{NaCl}, 10 \mathrm{mM}$ Tris- $\mathrm{HCl}$ at $\mathrm{pH}$ 7.5, $10 \mathrm{mM}$ EDTA, $0.5 \mathrm{mM}$ PMSF, $1 \mathrm{mM}$ DTT, $1 \times$ protease inhibitor cocktail [Calbiochem], and 400 units/ml RNase inhibitor), followed by incubation on ice for 10 minutes. After removing insoluble materials by centrifugation, lysates were precleared using $10 \mu \mathrm{l}$ of protein $\mathrm{G}$ sepharose $(\mathrm{NaCl}$ was added to a final concentration of $200 \mathrm{mM})$, followed by addition of yeast transfer RNA (tRNA) (Ambion) to a final concentration of $40 \mu \mathrm{g} / \mathrm{ml}$. The cleared lysates were transferred to tubes containing antibody or preimmune IgG-coated beads, and RIP was carried out by rotating the tubes at $4^{\circ} \mathrm{C}$ for 3 hours. Following RIP, the beads were washed $5 \times$ with IP buffer by adding $1 \mathrm{ml}$ of the buffer and rotating the tube at $4^{\circ} \mathrm{C}$ for 2 minutes each time. RNA was extracted from the beads using PureLink RNA Mini Kit (Ambion, 12183018A). Reverse transcription was performed in a $40 \mu \mathrm{l}$ reaction volume using PrimeScriptRT Reagent Kit (TAKARA Bio USA Inc., RR037A), followed by qPCR.

Glucose output assay. Glucose output assay was performed using Amplex Red Glucose/Glucose Oxidase Assay Kit (Molecular Probes, Invitrogen, A22189), according to the manufacturer's instructions. Briefly, HepG2 cells were transfected with siCon or siH19 in a 24-well plate, followed by glucose output assay 48 hours later. For primary mouse hepatocytes, cells were infected with AAV-Vec or AAV-H19 in a 12-well plate, and glucose output assay was carried out at 72 hours after infection. On the day of the assay, culture medium was replaced with glucose-free and phenol red-free DMEM (Gibco, A14430-01) for HepG2 or replaced with the same DMEM supplemented with $2 \mathrm{mM}$ L-glutamine and $15 \mathrm{mM}$ HEPES for primary hepatoctyes for 2 hours. Then, cells were incubated in $120 \mu 1$ glucose production medium (glucose-free and phenol red-free DMEM, $20 \mathrm{mM}$ sodium lactate, $2 \mathrm{mM}$ sodium pyruvate, and $0.5 \%$ BSA) for HepG 2 or in the same medium supplemented with $2 \mathrm{mM}$ L-glutamine and $15 \mathrm{mM}$ HEPES for primary hepatocytes for 4 hours. Then, $50 \mu \mathrm{l}$ of supernatant was used for measurement of glucose concentration, which was normalized to total protein content of cells.

QMSP. Genomic DNA was extracted from liver tissue samples or HepG2 cells in 1 well of 24-well plates using Quick-gDNA MicroPrep (ZR, D3021) according to the manufacturer's instructions. For bisulfite treatment, $200 \mathrm{ng}$ of DNA was used for each column using EZ DNA Methylation-Gold Kit (ZR, D5006). Elution buffer ( $100 \mu 1)$ was used to elute DNA from each column. qPCR was performed in a $15-\mu 1$ reaction containing $5 \mu \mathrm{l}$ of the eluant using iQSYBRGreen (Bio-Rad, 1708880) in a Bio-Rad iCycler. Two sets of PCR primers were designed: one for unmethylated and one for methylated DNA sequences. The PCR primers for methylated DNA were used at a final concentration of $0.6 \mu \mathrm{M}$ in each PCR reaction. PCR was performed by initial denaturation at $95^{\circ} \mathrm{C}$ for 5 minutes, followed by 40 cycles of 30 seconds at $95^{\circ} \mathrm{C}$, 30 seconds at $60^{\circ} \mathrm{C}$, and 30 seconds at $72^{\circ} \mathrm{C}$. Specificity was verified by melting curve analysis and agarose gel electrophoresis. The $\mathrm{Ct}$ values of each sample were used in the post-PCR data analysis. The relative levels of methylated versus unmethylated DNA sequences are presented in Figure 4B, Figure 5D, and Figure 6, A, D, and G. The primers used for QMSP are listed in Supplemental Table 1. 
Statistics. Statistical analyses and figure construction were performed using GraphPad Prism version 7.01 for Windows (GraphPad Software, www.graphpad.com). All data were analyzed using 2-tailed Student $t$ test and presented as mean \pm SEM. $P$ values at 0.05 or smaller were considered statistically significant.

Study approval. All animal work was approved by the Yale University IACUC.

\section{Author contributions}

NZ, TG, and YH designed the research and wrote the paper. NZ, TG, ZW, RZ, TC, and YL performed the experiments, analyzed the data, and prepared the figures. GIS and JPC designed and performed the hyperinsulinemic/euglycemic clamp studies. LD provided the H19-KO mice, SYC provided mouse primary hepatocytes, and HST and GGC provided intellectual insights and critical discussion of the project.

\section{Acknowledgments}

This work was supported by grants 1-15-BS-084 from the American Diabetes Association, GE001347 from the Mckern Albert foundation (both to YH), and R01 HD072418 from the NIH to (GGC). YL was supported by a fellowship 201608340011 from China Scholarship Council.

Address correspondence to: Yingqun Huang, Yale University School of Medicine, Department of Obstetrics, Gynecology \& Reproductive Sciences, 310 Cedar Street, LSOG 205C, New Haven, Connecticut 06510, USA. Phone: 203.737.2578; Email: Yingqun.huang@yale.edu.

1. Rines AK, Sharabi K, Tavares CD, Puigserver P. Targeting hepatic glucose metabolism in the treatment of type 2 diabetes. Nat Rev Drug Discov. 2016;15(11):786-804.

2. Hatting M, Tavares CDJ, Sharabi K, Rines AK, Puigserver P. Insulin regulation of gluconeogenesis. Ann N Y Acad Sci. 2018;1411(1):21-35

3. Yoon JC, et al. Control of hepatic gluconeogenesis through the transcriptional coactivator PGC-1. Nature. 2001;413(6852):131-138.

4. Rhee J, et al. Regulation of hepatic fasting response by PPARgamma coactivator-1alpha (PGC-1): requirement for hepatocyte nuclear factor 4alpha in gluconeogenesis. Proc Natl Acad Sci USA. 2003;100(7):4012-4017.

5. Zhang M, et al. Sodium caprate augments the hypoglycemic effect of berberine via AMPK in inhibiting hepatic gluconeogenesis. Mol Cell Endocrinol. 2012;363(1-2):122-130.

6. Souza Pauli LS, et al. Exercise training decreases mitogen-activated protein kinase phosphatase-3 expression and suppresses hepatic gluconeogenesis in obese mice. J Physiol (Lond). 2014;592(6):1325-1340.

7. Wei S, et al. Berberine Attenuates Development of the Hepatic Gluconeogenesis and Lipid Metabolism Disorder in Type 2 Diabetic Mice and in Palmitate-Incubated HepG2 Cells through Suppression of the HNF-4 $\alpha$ miR122 Pathway. PLoS One. 2016;11(3):e0152097.

8. Gabory A, Jammes H, Dandolo L. The H19 locus: role of an imprinted non-coding RNA in growth and development. Bioessays. 2010;32(6):473-480

9. Zhou J, et al. H19 lncRNA alters DNA methylation genome wide by regulating S-adenosylhomocysteine hydrolase. Nat Commun. 2015;6:10221.

10. Zhong T, et al. Metformin alters DNA methylation genome-wide via the H19/SAHH axis. Oncogene. 2017;36(17):2345-2354.

11. Zhang Y, et al. Bcl2 is a critical regulator of bile acid homeostasis by dictating Shp and lncRNA H19 function. Sci Rep. 2016;6:20559.

12. Nilsson E, et al. Epigenetic Alterations in Human Liver From Subjects With Type 2 Diabetes in Parallel With Reduced Folate Levels. J Clin Endocrinol Metab. 2015;100(11):E1491-E1501.

13. Yan Z, Yan H, Ou H. Human thyroxine binding globulin (TBG) promoter directs efficient and sustaining transgene expression in liver-specific pattern. Gene. 2012;506(2):289-294.

14. Bell P, et al. Effects of Self-Complementarity, Codon Optimization, Transgene, and Dose on Liver Transduction with AAV8 Hum Gene Ther Methods. 2016;27(6):228-237.

15. Stanya KJ, et al. Direct control of hepatic glucose production by interleukin-13 in mice. J Clin Invest. 2013;123(1):261-271.

16. Kim DH, et al. FoxO6 integrates insulin signaling with gluconeogenesis in the liver. Diabetes. 2011;60(11):2763-2774.

17. Ripoche MA, Kress C, Poirier F, Dandolo L. Deletion of the H19 transcription unit reveals the existence of a putative imprinting control element. Genes Dev. 1997;11(12):1596-1604.

18. Martinet C, Monnier P, Louault Y, Benard M, Gabory A, Dandolo L. H19 controls reactivation of the imprinted gene network during muscle regeneration. Development. 2016;143(6):962-971.

19. Si-Tayeb K, Lemaigre FP, Duncan SA. Organogenesis and development of the liver. Dev Cell. 2010;18(2):175-189.

20. Pope C, Mishra S, Russell J, Zhou Q, Zhong XB. Targeting H19, an Imprinted Long Non-Coding RNA, in Hepatic Functions and Liver Diseases. Diseases. 2017;5(1):E11.

21. He L, et al. Activation of the cAMP-PKA pathway Antagonizes Metformin Suppression of Hepatic Glucose Production. J Biol Chem. 2016;291(20):10562-10570.

22. Cai X, Cullen BR. The imprinted H19 noncoding RNA is a primary microRNA precursor. RNA. 2007;13(3):313-316.

23. Keniry A, et al. The H19 lincRNA is a developmental reservoir of miR-675 that suppresses growth and Igf1r. Nat Cell Biol. 2012;14(7):659-665. 
24. Perry RJ, et al. Hepatic acetyl CoA links adipose tissue inflammation to hepatic insulin resistance and type 2 diabetes. Cell. 2015;160(4):745-758

25. Sharabi K, et al. Selective Chemical Inhibition of PGC-1 $\alpha$ Gluconeogenic Activity Ameliorates Type 2 Diabetes. Cell. 2017;169(1):148-160.e15

26. Mutel E, et al. Control of blood glucose in the absence of hepatic glucose production during prolonged fasting in mice: induction of renal and intestinal gluconeogenesis by glucagon. Diabetes. 2011;60(12):3121-3131.

27. Penhoat A, Fayard L, Stefanutti A, Mithieux G, Rajas F. Intestinal gluconeogenesis is crucial to maintain a physiological fasting glycemia in the absence of hepatic glucose production in mice. Metab Clin Exp. 2014;63(1):104-111.

28. Baek SJ, et al. Integrated epigenomic analyses of enhancer as well as promoter regions in gastric cancer. Oncotarget. 2016;7(18):25620-25631.

29. Goyal N, et al. RNA sequencing of $\mathrm{db} / \mathrm{db}$ mice liver identifies lncRNA H19 as a key regulator of gluconeogenesis and hepatic glucose output. Sci Rep. 2017;7(1):8312.

30. Voutilainen R, Ilvesmäki V, Ariel I, Rachmilewitz J, de Groot N, Hochberg A. Parallel regulation of parentally imprinted H19 and insulin-like growth factor-II genes in cultured human fetal adrenal cells. Endocrinology. 1994;134(5):2051-2056.

31. Liu J, Kahri AI, Heikkilä P, Ilvesmäki V, Voutilainen R. H19 and insulin-like growth factor-II gene expression in adrenal tumors and cultured adrenal cells. J Clin Endocrinol Metab. 1995;80(2):492-496.

32. Unger RH, Aguilar-Parada E, Müller WA, Eisentraut AM. Studies of pancreatic alpha cell function in normal and diabetic subjects. J Clin Invest. 1970;49(4):837-848.

33. Godoy-Matos AF. The role of glucagon on type 2 diabetes at a glance. Diabetol Metab Syndr. 2014;6(1):91.

34. Ghazal S, et al. H19 lncRNA alters stromal cell growth via IGF signaling in the endometrium of women with endometriosis. EMBO Mol Med. 2015;7(8):996-1003.

35. Toda C, Kim JD, Impellizzeri D, Cuzzocrea S, Liu ZW, Diano S. UCP2 Regulates Mitochondrial Fission and Ventromedial Nucleus Control of Glucose Responsiveness. Cell. 2016;164(5):872-883.

36. Jurczak MJ, et al. Dissociation of inositol-requiring enzyme (IRE1 $\alpha$ )-mediated c-Jun N-terminal kinase activation from hepatic insulin resistance in conditional X-box-binding protein-1 (XBP1) knock-out mice. J Biol Chem. 2012;287(4):2558-2567.

37. Cai SY, et al. Bile acids initiate cholestatic liver injury by triggering a hepatocyte-specific inflammatory response. JCI Insight. 2017;2(5):e90780. 